Original Article

\title{
Incidence, risk factors, and CT characteristics of radiation recall pneumonitis induced by immune checkpoint inhibitor in lung cancer
}

\author{
François Cousin ${ }^{\mathrm{a}, *}$, Colin Desir $^{\mathrm{b}}$, Selma Ben Mustapha ${ }^{\mathrm{c}}$, Carole Mievis ${ }^{\mathrm{c}}$, Philippe Coucke ${ }^{\mathrm{c}}$, Roland Hustinx ${ }^{\mathrm{a}}$ \\ ${ }^{a}$ Department of Nuclear Medicine and Oncological Imaging; ${ }^{\mathrm{b}}$ Department of Radiology; and ${ }^{\mathrm{c}}$ Department of Radiotherapy, University Hospital (CHU) of Liège, Liège, Belgium
}

\section{A R T I C L E I N F O}

\section{Article history:}

Received 18 November 2020

Received in revised form 30 December 2020

Accepted 2 January 2021

Available online 14 January 2021

\section{Keywords:}

Lung cancer

Anti PD-1/PD-L1

Radiation pneumonitis

Radiation recall pneumonitis

\begin{abstract}
A B S T R A C T
Background and purpose: Radiation recall pneumonitis (RRP) is a delayed radiation-induced lung toxicity triggered by systemic agents, typically anticancer drugs. Immune checkpoint inhibitors (ICIs) have recently been identified as potential causal agents of RRP but its real incidence and potential risk factors remain unknown.

Materials and methods: Medical records and CTs of patients treated with programmed death 1 (PD-1) or programmed death ligand 1 (PD-L1) inhibitors for advanced lung cancer between 2014 and 2019 at our tertiary center, and who had a previous history of lung irradiation were retrospectively analyzed. We identified RRP as lung CT modifications occurring in the irradiation field $>6$ months after conventionally fractionated radiotherapy completion and $>1$ year after stereotactic body radiation therapy. Clinical and dosimetric data were analyzed to identify potential risk factors for RRP.

Results: Among 348 patients treated with ICIs, data from 80 eligible patients were analyzed (median age, 69 years [interquartile range, 11]; 45 men). Fifteen patients (18.8\%) presented with RRP. Median time between end of radiotherapy and RRP was 450 days (range, 231-1859). No risk factor was significantly associated with RRP. ICI-related pneumonitis was associated with RRP in $33.3 \%$ of cases $(p=0.0021)$, developing either concomitantly or after RRP. Incidence of grade $\geq 3$ pneumonitis in the RRP population was $13.3 \%$.

Conclusion: We demonstrated a high incidence of RRP (18.8\%) in our population of previously irradiated patients treated with ICIs for lung cancer. We identified no risk factors for RRP, but an association was noted between RRP and ICI-related pneumonitis.
\end{abstract}

(C) 2021 Elsevier B.V. All rights reserved. Radiotherapy and Oncology 157 (2021) 47-55
Radiation recall describes an unpredictable inflammatory reaction within previously irradiated tissue. It is triggered by pharmacological agents, typically anticancer drugs.

This phenomenon was first described in 1959 by D'Angio et al. who reported the reactivation of latent radiation dermatitis after actinomycin D therapy [1]. It has since been observed in many other tissues such as muscles, bowel, central nervous system and lungs [2-5]. This poorly understood reaction differs from the classical radiation-induced toxicity in the sense that it occurs typically tardily, sometimes several years after irradiation [6]. Radiation recall pneumonitis (RRP), has been subject to particular concern because lesions visible on lung CT can be extensive, sometimes

\footnotetext{
Abbreviations: RRP, radiation recall pneumonitis; ICI, immune checkpoint inhibitor; PD-1, programmed death 1; PD-L1, programmed death ligand 1; OR, overall survival; PFS, progression-free survival; SBRT, stereotactic body radiation therapy; iRECIST, immune Response Evaluation Criteria in Solid Tumors.

* Corresponding author at: Avenue de l'hôpital 1, 4000 Liège, Belgium.

E-mail address: fcousin@chuliege.be (F. Cousin).
}

associated with severe symptoms leading to treatment discontinuation.

Anticancer molecules most commonly involved with RRP are classical chemotherapeutic agents, such as taxanes, gemcitabine, and targeted therapies such as tyrosine kinase inhibitors [5,7]. More recently, several cases of radiation recall pneumonitis associated with immune checkpoint inhibitors (ICIs) have also been described $[6,8,9]$. In the last decade, ICIs have demonstrated unprecedented efficacy in the treatment of many advanced malignancies such as lung cancer [10]. However, as ICIs act by harnessing the immune system in order to limit tumor proliferation, they have been associated with a panel of specific inflammatory adverse events called immune-related adverse events, among which pneumonitis $[11,12]$. With the increasing use of ICIs in advanced lung cancer, we can expect that previously irradiated patients will be more exposed to radiation recall phenomenon, especially to the lungs due to the combined toxicity. Occurrence of such abnormalities on lung CT in a patient during follow-up under ICIs is always challenging. Therefore, a deeper understanding of RRP and its early 
recognition on imaging are both crucial. Yet, little is known about incidence and risk factors for RRP in the setting of immunotherapy [13].

We therefore conducted a retrospective study to assess the incidence, the clinical and radiological characteristics and the potential risk factors of RRP in a population of patients with lung cancer treated with programmed death 1 (PD-1) or anti-programmed death-ligand 1 (PD-L1) inhibitors.

\section{Materials and methods}

\section{Patients}

We retrospectively reviewed the medical, radiological and radiation therapy records of all patients treated with PD-1/PD-L1 inhibitors for advanced lung cancer between 2015 and 2019 at our institution. Patients were included in our study if they had received thoracic irradiation (either to the lung, the mediastinum, the thoracic wall or the breast), of any type, with a minimum total dose of $15 \mathrm{~Gy}$ (pneumonitis is not expected to occur with smaller doses) $[14,15]$. Radiotherapy had to be terminated before initiation of immunotherapy to avoid cumulative effects. Patients who had received concurrent ICIs and radiotherapy, or ICIs before radiotherapy, were excluded. Patients who had the irradiated lung area surgically removed before ICI initiation were excluded. Patients who received a maintenance treatment with durvalumab following thoracic chemoradiation were not included because they represent a very specific population with a high risk of lung toxicity due to treatments cumulative effects.

The following demographic and clinical data, summarized in Table 1, were collected: age, sex, smoking history, presence of lung emphysema or interstitial lung disease assessed by imaging prior to ICI initiation, overall survival (OS), progression-free survival (PFS), tumor histology, tumoral PD-L1 expression, ICI molecule, prior chemotherapy and development of an ICI-related pneumonitis.

PFS was defined as the time from the first dose of ICI to disease progression according to immune Response Evaluation Criteria in Solid Tumors (iRECIST), or death from any cause.

OS was defined as the time from the first dose of ICI to the date of death from any cause.

The diagnosis of ICI-related pneumonitis was determined in the multidisciplinary tumor board, including at least a pulmonologist, an oncologist and a radiologist and was based on the occurrence of

Table 1

Population characteristics.

\begin{tabular}{|c|c|c|c|c|}
\hline Characteristics & $\begin{array}{l}\text { No RRP } \\
(n=65)\end{array}$ & $\begin{array}{l}\text { RRP } \\
(n=15)\end{array}$ & $\begin{array}{l}\text { All } \\
(n=80)\end{array}$ & $p$ values \\
\hline Sex & & & & 0.37 \\
\hline Women, $n(\%)$ & $30(46)$ & $5(33)$ & $35(44)$ & \\
\hline Men, $n(\%)$ & $35(54)$ & $10(67)$ & $45(56)$ & \\
\hline Median age, y (IQR) & $67(11)$ & $70(10)$ & $69(11)$ & 0.33 \\
\hline Smoking history, $n(\%)$ & $62(95)$ & $14(93)$ & $76(95)$ & 0.57 \\
\hline Prior emphysema, $n$ (\%) & & & & 0.55 \\
\hline Absence & $20(31)$ & $4(27)$ & $24(30)$ & \\
\hline Mild & $17(26)$ & $6(40)$ & $23(29)$ & \\
\hline Moderate & $18(28)$ & $2(13)$ & $20(25)$ & \\
\hline Severe & $10(15)$ & $3(20)$ & $13(16)$ & \\
\hline Tumour histology, $n$ (\%) & & & & 0.27 \\
\hline Adenocarcinoma & $24(37)$ & $6(40)$ & $30(38)$ & \\
\hline Squamous & $31(48)$ & $6(40)$ & $37(46)$ & \\
\hline Other ${ }^{1}$ & $10(15)$ & $3(20)$ & $13(16)$ & \\
\hline ICI molecule, $n(\%)$ & & & & 0.58 \\
\hline Nivolumab & $43(66)$ & $10(67)$ & $53(66)$ & \\
\hline Pembrolizumab & $14(22)$ & $2(13)$ & $16(20)$ & \\
\hline Atezolizumab & $8(12)$ & $3(20)$ & $11(14)$ & \\
\hline Prior chemotherapy, $n(\%)$ & $49(75)$ & $11(73)$ & $60(75)$ & 0.55 \\
\hline Concurrent CT-RT, $n(\%)$ & $26(40)$ & $8(53)$ & $34(43)$ & 0.89 \\
\hline Combined chemotherapy, $n(\%)$ & $3(5)$ & $1(7)$ & $4(5)$ & 0.57 \\
\hline \multicolumn{5}{|l|}{ Prior radiotherapy, $n(\%)$} \\
\hline Type & & & & 0.10 \\
\hline Palliative & $5(8)$ & $0(0)$ & $5(6)$ & \\
\hline \multicolumn{5}{|l|}{ Curative } \\
\hline Conventional & $49(75)$ & $12(80)$ & $61(76)$ & \\
\hline SBRT & $11(17)$ & $3(20)$ & $14(18)$ & \\
\hline Median radiation dose, Gy (IQR) & & & & 0.58 \\
\hline Conventional & $66(9)$ & $60(16.5)$ & $66(12)$ & \\
\hline SBRT & $60(7.5)$ & $60(5)$ & $60(10)$ & \\
\hline Palliative & $17(0)$ & 1 & $17(0)$ & \\
\hline Median dose/fraction, Gy (IQR) & & & & 0.86 \\
\hline Conventional & $2(0)$ & $2(0.5)$ & $2(0.1)$ & \\
\hline SBRT & $20(10)$ & $12(5)$ & $16(10)$ & \\
\hline Palliative & $8.5(0)$ & 1 & $8.5(0)$ & \\
\hline Median MLD, Gy (IQR) & $10.2(9.7)$ & $11(9.2)$ & $10.5(9.3)$ & 0.84 \\
\hline Median V20, \% (IQR) & $16.1(16.8)$ & $19.6(17.4)$ & $17.7(16.8)$ & 0.66 \\
\hline Median time between end of RT and ICI initiation, $d$ (IQR) & $388(524)$ & 320 (499) & $368.5(529)$ & 0.89 \\
\hline ICI-related pneumonitis, $n(\%)$ & $2(3)$ & $5(33)$ & $7(9)$ & 0.002 \\
\hline Median PFS, $m$ (IQR) & $235(347)$ & $324(291)$ & $262(340)$ & 0.99 \\
\hline Median OS, $m$ (IQR) & $384(369)$ & $376(535)$ & $384(392)$ & 0.84 \\
\hline
\end{tabular}

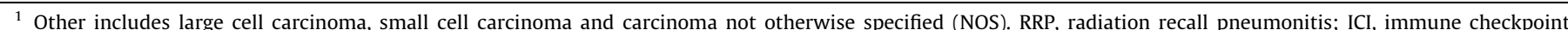

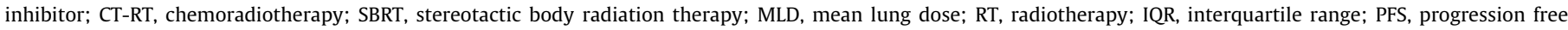
survival; OS, overall survival. 
diffuse lung abnormalities on CT, after exclusion of other alternative etiologies such as infection or tumor progression. The following radiation parameters were collected: type of radiotherapy (palliative, conventionally fractionated or stereotactic body radiation therapy (SBRT)), region targeted, total dose, dose per fraction, mean lung dose, V20 (volume of lung receiving $20 \mathrm{~Gy}$ ).

This study received approval from our institutional review board, and the need for informed consent was waived based on its retrospective design.

\section{CT analysis}

Radiological follow-up by lung CT, with or without contrast media, of at least 6 months after conventionally fractionated radiotherapy and 1 year after SBRT was needed for inclusion. Patients were also required to have had at least one lung CT prior to ICI initiation (baseline $\mathrm{CT}$ ) and at least one lung CT carried out after ICI initiation.

All CTs (baseline CTs and follow up CTs under ICIs) were reviewed in consensus by two board-certified radiologists who were unaware of the initial report (F.C. and C.D, with respectively 5 and 1 years of experience in thoracic imaging).

RRP was defined, in opposition to ICI-related pneumonitis, as the appearance of typical radiological changes of radiation pneumonitis on CT (homogeneous or patchy areas of ground glass opacity or consolidation, evolving progressively to retractile and fibrotic changes over time) developing within the radiation field later than 6 months after conventionally fractionated radiotherapy and later than 1 year after SBRT [16,17]. All new lung modifications were included, regardless of the presence of preexisting radiationinduced lung fibrosis. Pulmonary infection was ruled out by bronchoscopy microbiology and/or clinical course. Tumor recurrence was distinguished from RRP based on radiological criteria and/or long term clinical and radiological follow up. Occurrence time of RRP was defined as the time between radiotherapy completion date and the date of the $\mathrm{CT}$ on which first lung modifications were seen. The severity of the pneumonitis was evaluated using the Common Terminology Criteria for Adverse Events v 5.0 grades. The presence of respiratory symptoms related to RRP was also assessed.

The following radiological characteristics concerning RRP were assessed on CT: presence of prior radiation-induced lung fibrosis (consolidation with volume-loss, parenchymal distortion and traction bronchiectasis), presence of ground glass opacities and/or consolidations, distribution of lung changes within the irradiation field (diffuse or patchy). We retrospectively reviewed the initial radiological reports of the CTs of patients with RRP and the diagnoses made at that time were analyzed.

\section{Statistics}

Results are expressed as numbers and frequencies for qualitative parameters and as mean and standard deviation, median and interquartile range and range for quantitative parameters. The normality of the quantitative parameters was investigated using the mean-median comparison, the histogram and Quantile-Quantile plot and the Shapiro-Wilk test. Comparison between the two groups (patients with or without RRP) was assessed using a Chisquared test (or Fisher's exact test) for qualitative risk factors and the Mann-Whitney test for quantitative risk factors. Comparison of RRP grade between groups was assessed using a KruskalWallis test. Correlations between RRP grade and quantitative risk factors were calculated and tested with a Spearman correlation test.
Statistical significance was set at $p<0.05$. All statistical analyses were performed using SAS v 9.4 software (SAS Institute, Cary, NC).

\section{Results}

A total of 348 patients with advanced lung cancer treated with PD-1/PD-L1 inhibitors were identified, of whom 112 had prior lung irradiation. Thirty-two patients were excluded due to either insufficient CT follow-up, irradiation dose to the lung less than $15 \mathrm{~Gy}$ or surgical excision of the irradiated lobe (Fig. 1). A total of 80 patients were included in our statistical analysis (median age, 69 years [interquartile range, 11]; 45 men). Demographic characteristics of the population are presented in Table 1. PD-L1 status of the tumor was available only for 30 (37.5\%) patients and was therefore not included in our statistical analysis.

The median time between completion of radiotherapy and initiation of immunotherapy was 368.5 days (range, 10-4864). Fifteen out of $80(18.8 \%)$ patients presented with RRP. Demographic, clinical and radiological characteristics of the 15 RRP patients are shown in Tables 2 and 3. The median time between completion of radiotherapy and RRP was 450 days (range, 231-1859 days) and the median time between initiation of immunotherapy and the occurrence of RRP was 61 days (range, 4-520 days). Twelve of 66 (18.2\%) patients who received conventionally fractionated radiotherapy presented radiation pneumonitis > 6 months after irradiation, among whom 7 (10.6\%) patients developed radiation pneumonitis $>1$ year after irradiation (Fig. 2). One patient presented lung modifications 5 years after radiotherapy completion. Of the 14 patients who received SBRT,

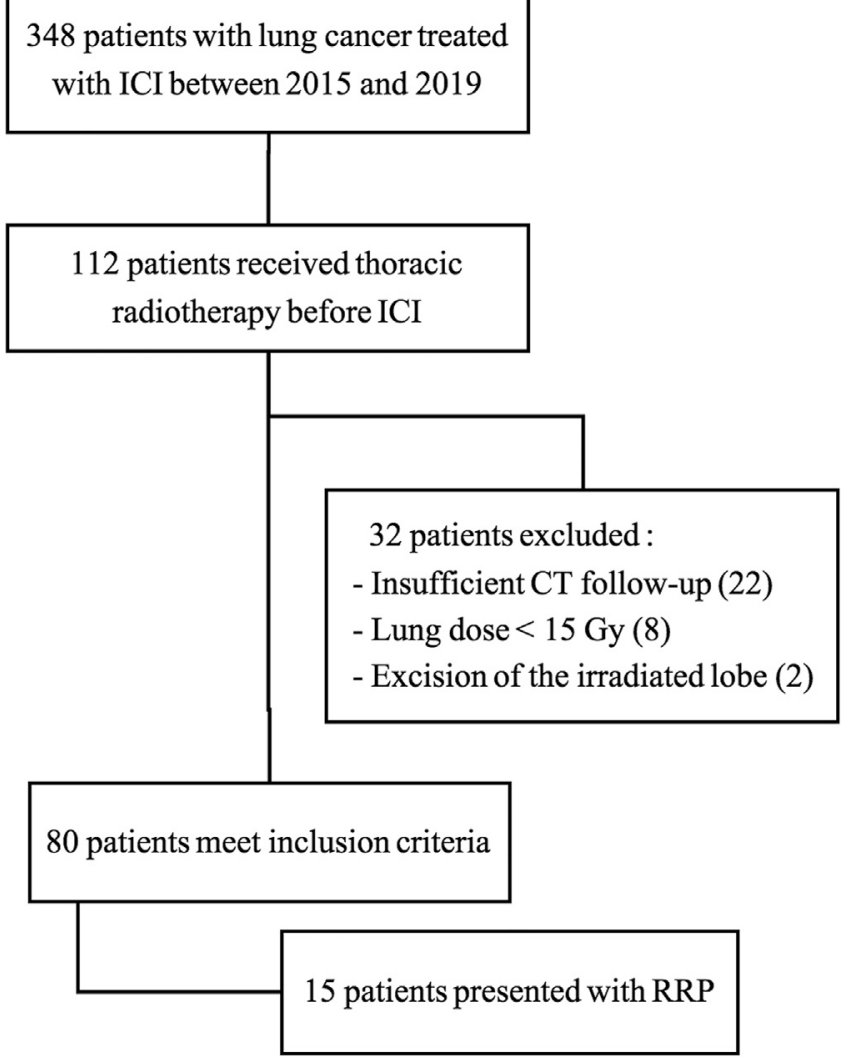

Fig. 1. Patients disposition. ICI, immune checkpoint inhibitor; RRP, radiation recall pneumonitis. 
Table 2

Characteristics of the RRP patients.

\begin{tabular}{ll}
\hline Variables & $n=15$ \\
\hline CTCAE grade, $n(\%)$ & $10(67)$ \\
1 & $3(20)$ \\
2 & $2(13)$ \\
3 & $0(0)$ \\
$>3$ & \\
CT characteristics, $n(\%)$ & $11(73)$ \\
$\quad$ Prior radiation fibrosis & $10(67)$ \\
Ground glass opacity & $13(87)$ \\
Consolidation & \\
RRP distribution & $5(33)$ \\
$\quad$ Patchy & $10(67)$ \\
$\quad$ Diffuse & $8(53)$ \\
Diagnosis on CT & \\
$\quad$ Tumoral progression & $6(40)$ \\
$\quad$ Radiation pneumonitis & $1(7)$ \\
$\quad$ Infection & $450(498)$ \\
Median time between radiotherapy and RPP, $d(\mathrm{IQR})$ & $61(90)$ \\
Median time between ICI and RRP, $d$ (IQR) &
\end{tabular}

${ }^{1}$ Corresponding to the initial diagnosis made on CT at the time of realization of the images. RRP, radiation recall pneumonitis; CTCAE, Common Terminology Criteria for Adverse Events; IQR, interquartile range; ICI, immune checkpoint inhibitor.

$3(21.4 \%)$ patients presented radiation pneumonitis > 1 year, among whom 1 patient presented radiation pneumonitis 2 years and 4 months after radiotherapy completion (Fig. 3). None of the 5 patients who received palliative radiotherapy presented with RRP. If we look at the whole population regardless of the type of irradiation received, 10 out of $80(12.5 \%)$ patients presented with radiation pneumonitis after 12 months.

The majority of cases of RRP were low grade, 2 of 15 (13.3\%) patients presented a grade $\geq 3$ pneumonitis and only 5 of 15 (33.3\%) patients with RRP presented with symptoms. One patient who presented RRP with grade 2 symptoms also presented with synchronous signs of ICI-related pneumonitis on CT (nonspecific interstitial pneumonia pattern) which could also have induced symptoms.

PFS and OR medians were not significantly different between patients with or without RRP (324 and 235 days, $p=0.99 ; 376$ and 384 days, $p=0.84$, respectively).
Among the 15 patients with RRP, 11 (73.3\%) patients demonstrated signs of prior radiation-induced lung fibrosis on baseline CT. RRP manifested on CT with ground glass opacity in 10 (66.7\%) patients and with consolidation in 13 (86.7\%) patients. A diffuse distribution of the lung abnormalities on CT was noted in 10 (66.7\%) patients as opposed to patchy appearance in 5 (33.3) patients (Fig. 4).

The reports that were generated at the time of realization of CT correctly identified RRP in only 6 of $15(40 \%)$ cases, RRP was mistaken for tumoral progression in 8 of 15 (53.3\%) cases and with infection in 1 of 15 (6.7 \%) cases.

Table 1 shows the association between patient-, disease-, and treatment-related variables and incidence of RRP. None of the following dosimetric factors was associated with RRP: the type of irradiation (palliative, normally fractionated or SBRT) $(p=.10)$, the lung site irradiated $(p=.50)$, the radiation dose $(p=.58)$, the dose per fraction $(p=.86)$, the MLD $(p=.84)$ and the V20 $(p=.66)$.

The only variable that was significantly associated with RRP was the presence of an ICI-related pneumonitis $(p=.0021): 5$ of 15 (33.3\%) patients with RRP also presented with ICI-related pneumonitis, of whom 2 patients presented RRP and ICI-related pneumonitis concurrently and 3 patients presented initially with RRP and later with ICI-related pneumonitis.

\section{Discussion}

Our study describes the incidence of, and risk factors for, the development of RRP in a population of previously irradiated patients treated with PD-1/PD-L1 inhibitors for advanced lung cancer. To the best of our knowledge, no observational study has evaluated RRP in the setting of immunotherapy before. We observed in our population an unexpectedly high incidence of RRP (18,8\%), occurring as late as 5 years after irradiation, without any identified risk factor.

Radiation-induced lung injury is generally divided temporally into two phases, an acute inflammatory phase called radiation pneumonitis occurring within 6 months after radiotherapy completion, followed by a late or fibrotic phase developing after 6 months [17].

After conventionally fractionated radiotherapy, the first radiological manifestations of radiation pneumonitis are expected to

Table 3

Individual characteristics of the 15 RRP patients.

\begin{tabular}{|c|c|c|c|c|c|c|c|c|c|c|c|c|c|}
\hline \multicolumn{3}{|c|}{ Demographics } & \multicolumn{3}{|c|}{ RT Treatment Characteristics } & \multicolumn{4}{|c|}{ RRP Clinical Characteristics } & \multicolumn{4}{|c|}{ RRP CT Characteristics } \\
\hline Age & Sex & $\begin{array}{l}\text { Smoking } \\
\text { history }\end{array}$ & Type of RT & $\begin{array}{l}\text { MLD } \\
\text { (Gy) }\end{array}$ & $\begin{array}{l}\text { V20 } \\
(\%)\end{array}$ & $\begin{array}{l}\text { Months } \\
\text { between end } \\
\text { of RT and RRP }\end{array}$ & $\begin{array}{l}\text { Months } \\
\text { between } \\
\text { start of ICI } \\
\text { and RRP }\end{array}$ & $\begin{array}{l}\text { Association } \\
\text { with IR } \\
\text { pneumonitis }\end{array}$ & $\begin{array}{l}\text { Grade } \\
\text { of RRP } \\
\text { (CTCAE) }\end{array}$ & GGO & Consolidation & Distribution & $\begin{array}{l}\text { Initial } \\
\text { diagnosis } \\
\text { based on } \\
\text { CT }\end{array}$ \\
\hline 78 & M & Yes & Conventional & 6.5 & 14.8 & 7 & 2 & Yes & 2 & No & Yes & Diffuse & RRP \\
\hline 72 & $\mathrm{~F}$ & Yes & Conventional & 14.1 & 26.8 & 11 & 1 & Yes & 1 & Yes & Yes & Patchy & RRP \\
\hline 78 & M & Yes & SBRT & 4.9 & 5.5 & 12 & 2 & No & 1 & No & Yes & Diffuse & Progression \\
\hline 68 & $\mathrm{~F}$ & Yes & Conventional & 15.7 & 26.3 & 10 & 1 & No & 1 & Yes & Yes & Patchy & Progression \\
\hline 69 & M & Yes & Conventional & 11.2 & 19.7 & 15 & 1 & No & 2 & Yes & Yes & Diffuse & RRP \\
\hline 73 & $\mathrm{~F}$ & No & Conventional & 4.8 & 8.9 & 40 & 27 & Yes & 1 & Yes & Yes & Diffuse & Progression \\
\hline 78 & M & Yes & Conventional & 9.1 & 17.5 & 12 & $<1$ & No & 1 & No & Yes & Diffuse & Progression \\
\hline 68 & M & Yes & SBRT & 2.4 & 1.9 & 15 & 4 & Yes & 3 & No & Yes & Diffuse & Progression \\
\hline 68 & M & Yes & Conventional & 16.4 & 27.1 & 17 & 2 & No & 1 & Yes & Yes & Patchy & Progression \\
\hline 64 & M & Yes & Conventional & 10.9 & 19.5 & 49 & 17 & No & 1 & No & Yes & Diffuse & RRP \\
\hline 77 & $\mathrm{~F}$ & Yes & Conventional & 12.6 & 22.5 & 14 & 4 & No & 1 & Yes & Yes & Patchy & RRP \\
\hline 63 & M & Yes & SBRT & 2.3 & 1 & 27 & 1 & Yes & 2 & Yes & Yes & Patchy & Progression \\
\hline 69 & $\mathrm{~F}$ & Yes & Conventional & 1 & 1 & 61 & $<1$ & No & 1 & Yes & No & Diffuse & Infection \\
\hline 63 & M & Yes & Conventional & 12.7 & 23.7 & 8 & 3 & No & 3 & Yes & Yes & Diffuse & Progression \\
\hline 55 & M & Yes & Conventional & 16.5 & 28.2 & 10 & 9 & No & 1 & Yes & No & Diffuse & RRP \\
\hline
\end{tabular}

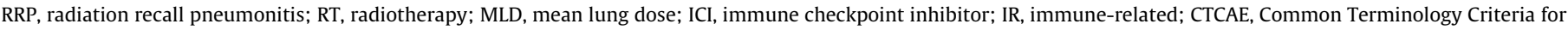
Adverse Events; GGO, ground glass opacity; SBRT, stereotactic body radiation therapy. 


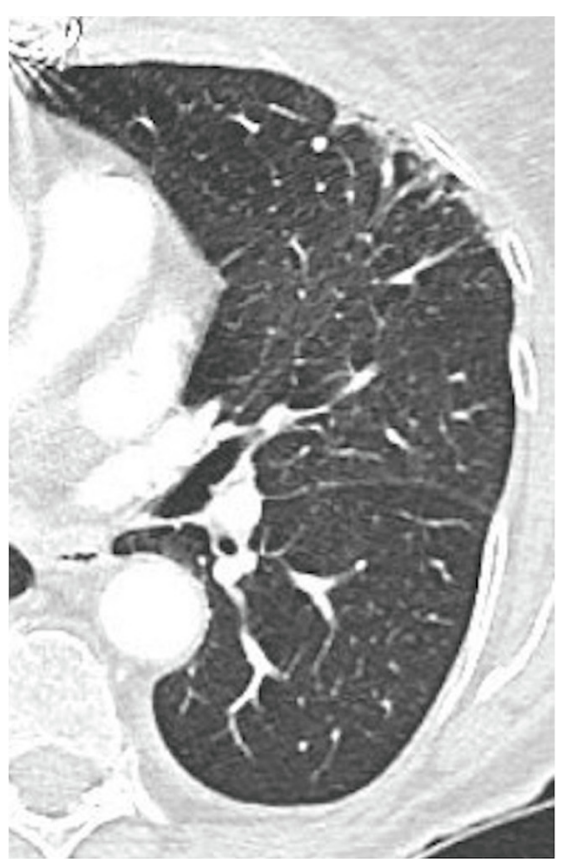

a

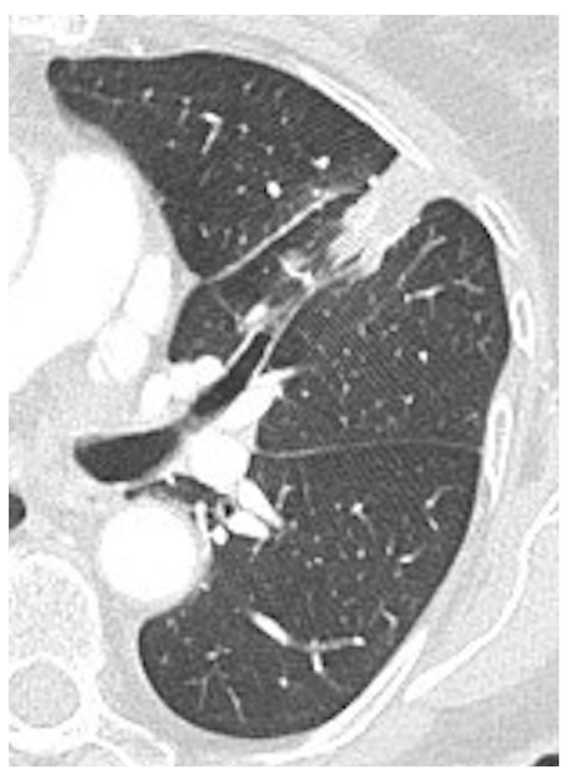

C

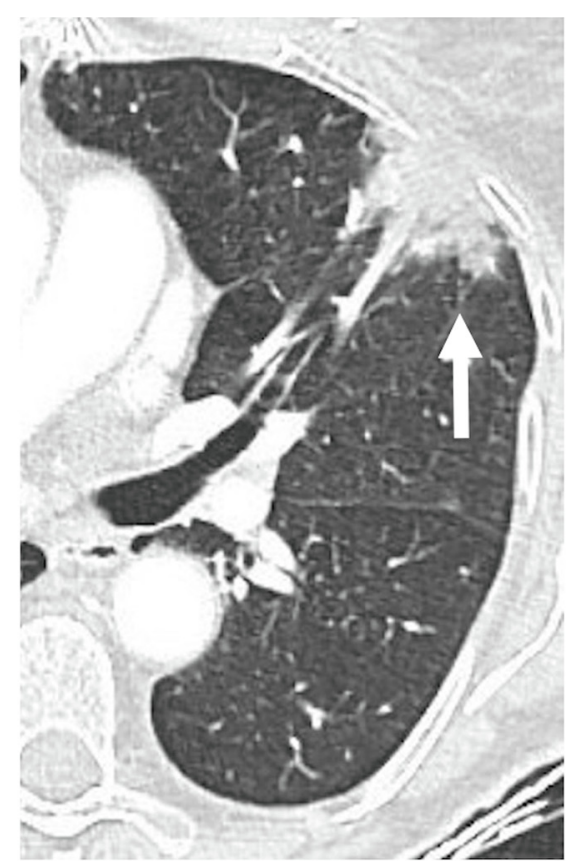

b

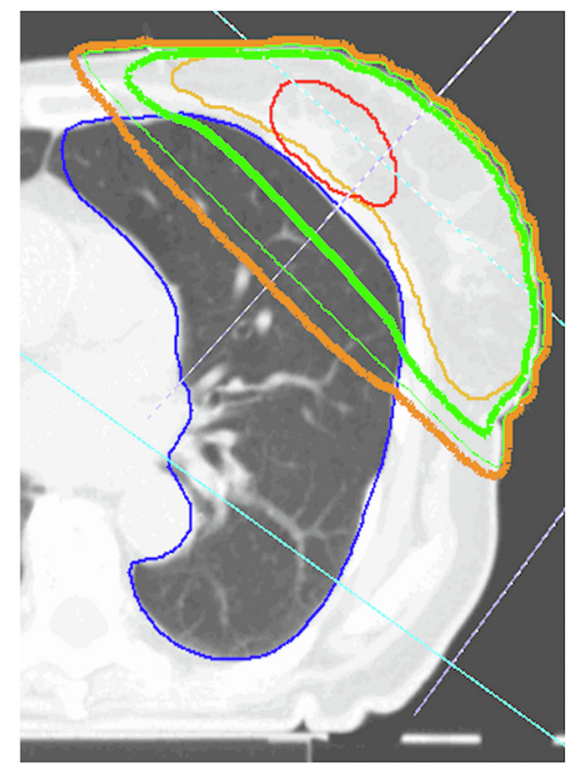

d

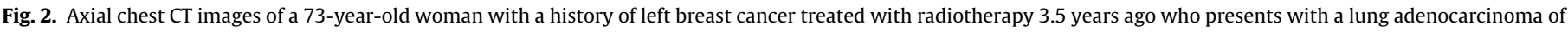

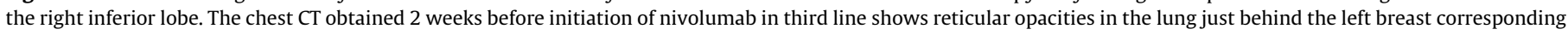

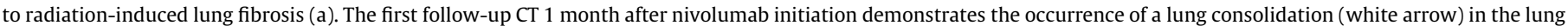

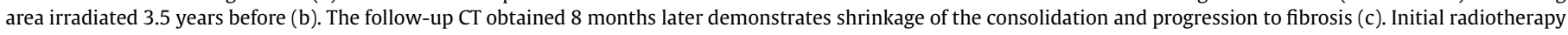
plan with bolded orange and green lines corresponding respectively to the $10 \mathrm{~Gy}$ and $40 \mathrm{~Gy}$ isodose lines (d).

occur around 3 to 4 months [15,18-20]. They correspond pathologically to an early inflammatory reaction of the lung secondary to cellular damages and is characterized radiologically by groundglass opacities and/or consolidation visible on CT in the irradiation field [16,21]. Concerning SBRT, lung modifications are also expected to occur within 6 months after radiotherapy completion but are typically delayed due to the higher dose per fraction, and could appear up to one year after irradiation [15,22-24].

Most of the cellular damage related to radiation repairs over time, but it is now recognized that changes in cellular function and inflammation pathways can persist over time [25]. Among these changes is the persistent damage to pulmonary stem cells. Irradiation can cause stress-induced senescence of stem cells in the lung parenchyma, making them unable to repair tissue damage and capable of enhancing inflammatory reaction through the secretion of cytokines and growth factors [26]. Long-term modifications in the T-cell population in irradiated lung, with an increased number and alteration of the balance of T-cell subsets due to distinct radiosensitivity, may also be implicated in radiation-induced lung injury and latent inflammatory processes 


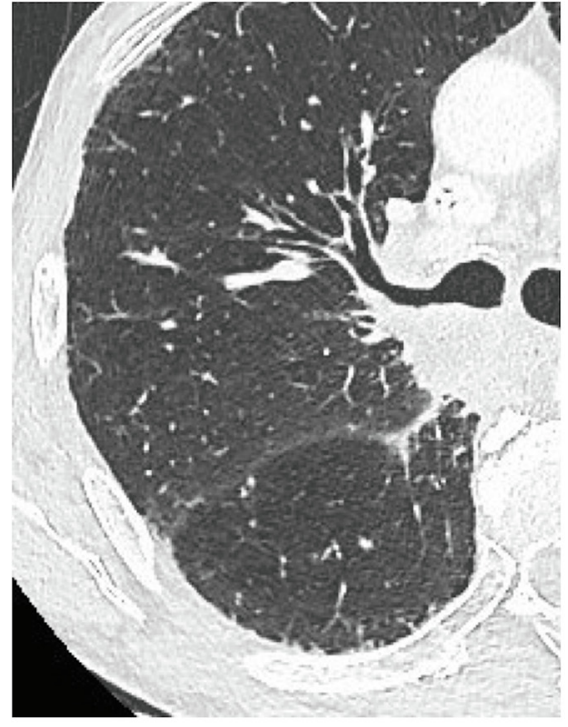

a

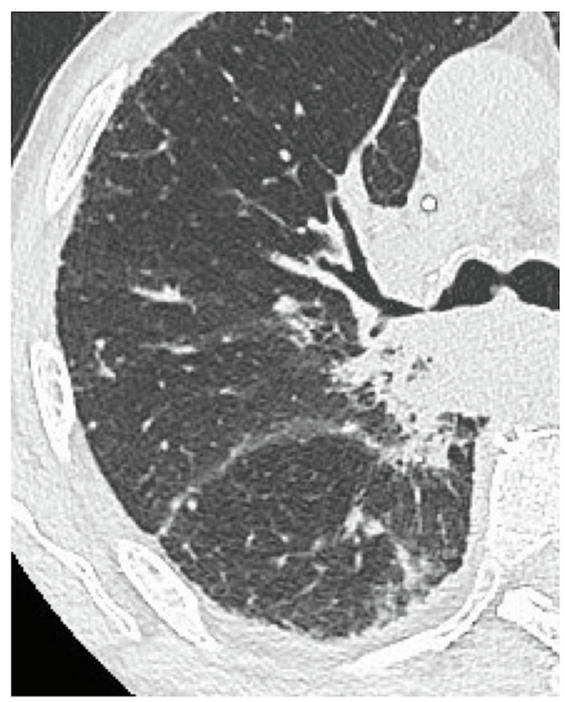

C

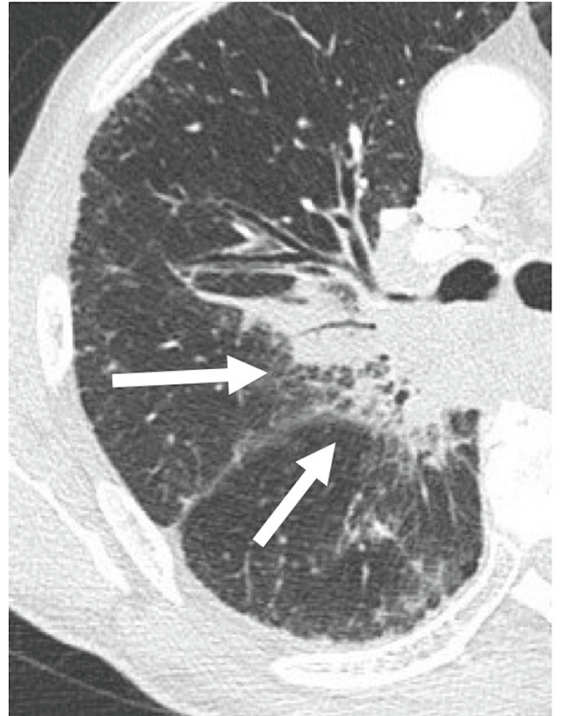

b

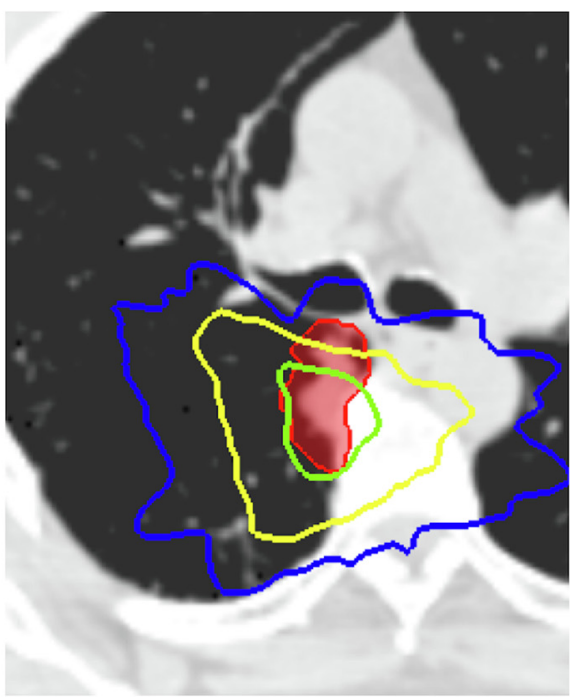

d

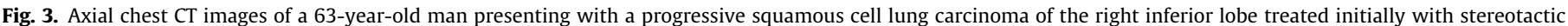

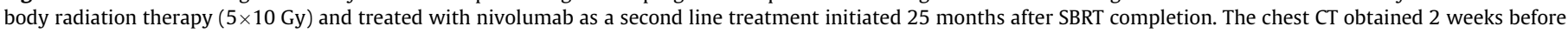

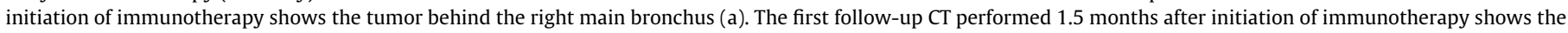

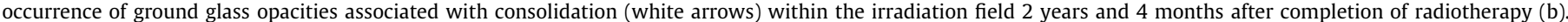

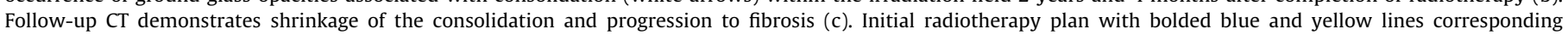
respectively to the $5 \mathrm{~Gy}$ and $16 \mathrm{~Gy}$ isodose lines (d).

$[25,27,28]$. Thus, lung parenchyma which has not reached the irradiation threshold to develop the chain reaction leading to pneumonitis, has yet experienced tissue injuries that sensitize it to inflammatory reaction. This realize a latent pro-inflammatory state, which can subsequently be reactivated, even years later.

Radiotherapy is now recognized as being an activator of several key elements of the local immune response and interactions between irradiation and immunotherapy are increasingly recognized [29]. Several studies have focused on identifying the association between the development of ICI-related pneumonitis and previous chest radiotherapy. On the one hand, some studies have shown no increase of all-grade pneumonitis in patients who have previously received thoracic radiotherapy compared to patients who have not $[30,31]$. On the other hand, in a secondary analysis of the KEYNOTE-001 phase 1 trial, Shaverdian et al. noticed that patients who previously received thoracic radiotherapy were more likely to have any-grade pulmonary toxicity, especially pneumonitis, suggesting the need for close toxicity monitoring in this subset of patients [32]. The same observation was made by Antonia et al. in the phase III PACIFIC trial in which they noticed a greater proportion of pneumonitis in the group of patients receiving durvalumab after chemoradiation for lung cancer compared to the placebo group (33.9\% versus 24.8\%) [33]. These results suggest the role of radiotherapy in priming the immune response and promoting immune-mediated lung toxicity.

Interestingly, none of these studies has clearly differentiated radiation pneumonitis from ICI-related pneumonitis. In our study, we identified radiation pneumonitis as typical radiological changes 


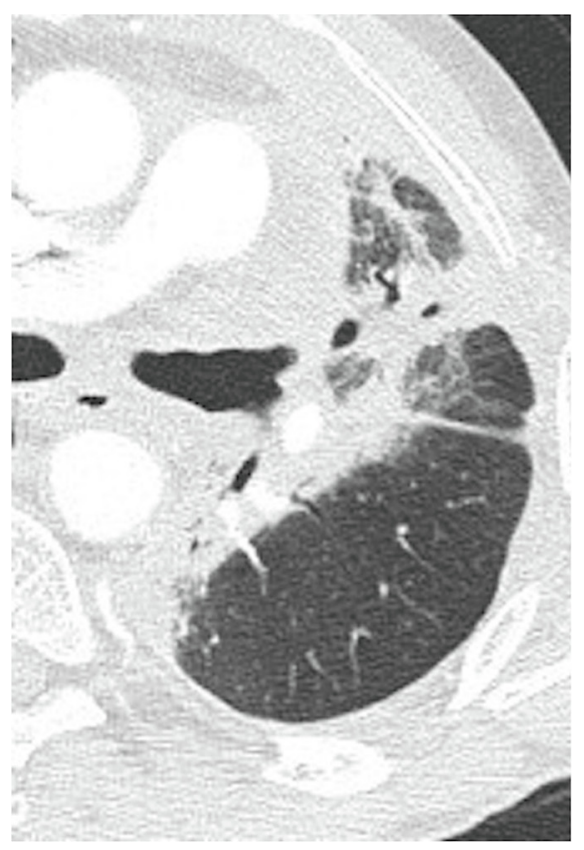

a

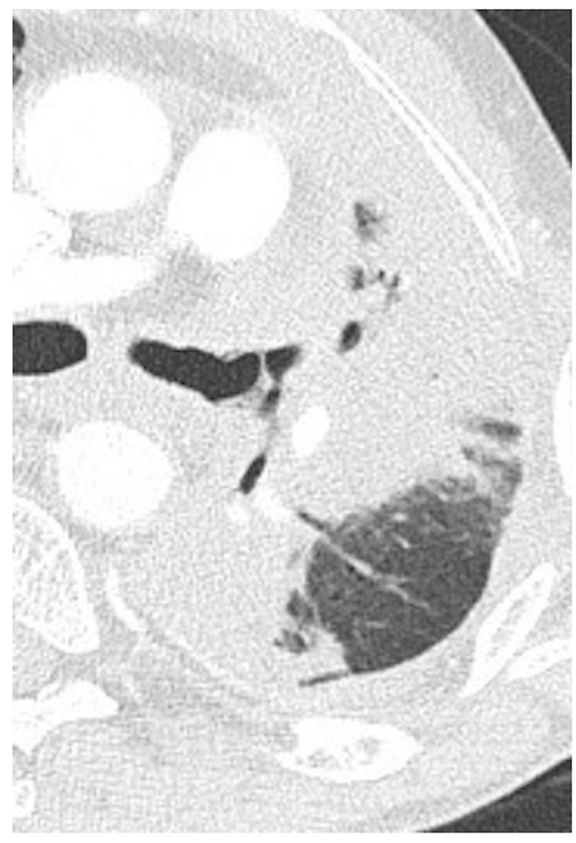

C

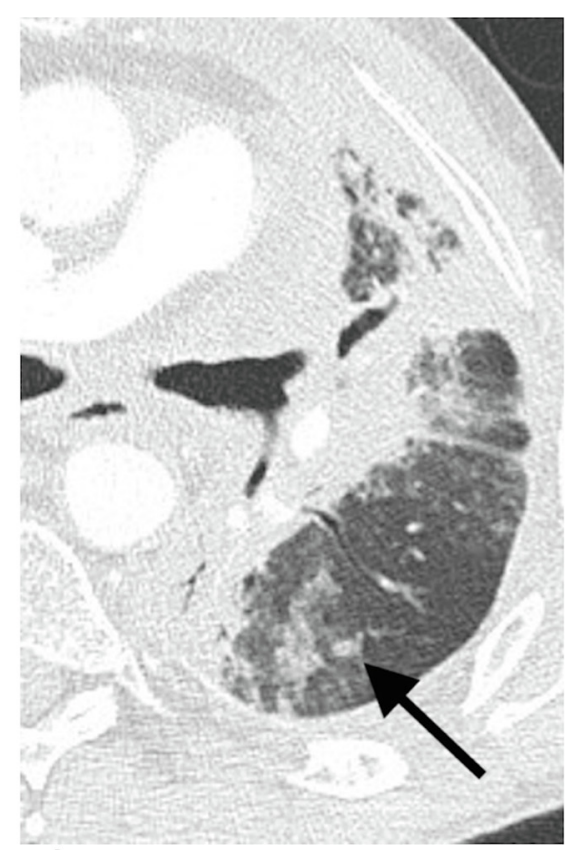

b

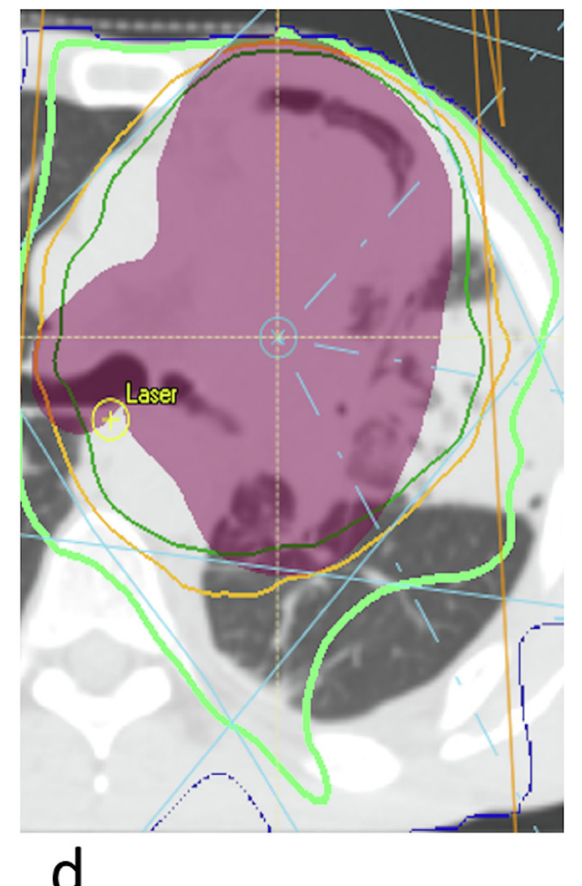

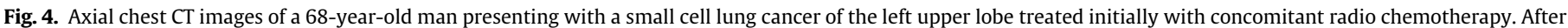

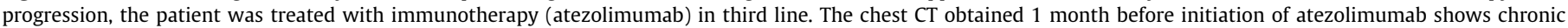

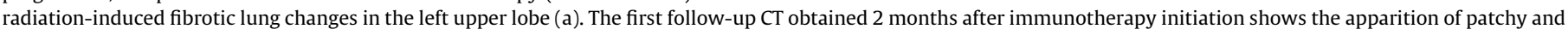

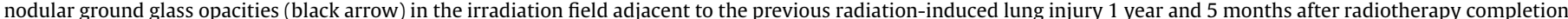

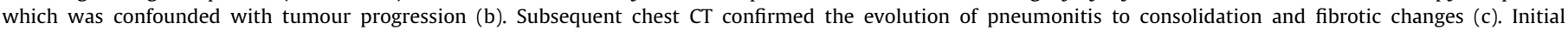
radiotherapy plan with isodose lines with the bolded green line corresponding to the $36 \mathrm{~Gy}$ isodose line (d).

located within the irradiation field, as opposed to ICI-related pneumonitis which does not present with a specific pattern but is typically diffuse and rarely evolves into fibrotic changes [12,34-36].

However, the question of whether our observations correspond to radiation pneumonitis or ICI-related pneumonitis occurring in the irradiation field is relevant. Pozzessere et al., who looked at a subset of 15 patients with ICI-related pneumonitis, noticed a pref- erential location of pneumonitis in the irradiation fields if patients had previously received radiotherapy. However, they ruled out radiation pneumonitis when it occurred $>6$ months after radiotherapy completion, despite their suggestion of a potential radiation recall effect [37]. As a matter of fact, both mechanisms probably overlap. In our population, we also noticed a significant association between radiation pneumonitis and ICI-related pneumonitis, with 
the latter occurring either at the same time, or later, than RRP. However, more studies are needed to gain a better understanding of these crossed mechanisms.

Of particular interest is our finding of highly delayed radiation pneumonitis in our population of patients treated with ICIs. Based on this observation, we suggest that the usual definition of radiation pneumonitis based on the onset time is no longer appropriate in the immunotherapy setting, and a period of time $>6$ months between radiotherapy and pneumonitis should not rule out the diagnosis of radiation pneumonitis. The recognition of radiation pneumonitis is of utmost importance in the context of oncological follow-up, especially if it appears tardily after radiotherapy completion because a causal link is therefore more difficult to establish.

Our study also presents some limitations. First, it is a retrospective single institution analysis. Additional prospective studies with larger sample sizes are required to confirm our observations. There is no consensus definition of RRP in the literature, and we arbitrarily defined RRP as changes appearing on CT $>6$ months after conventional radiotherapy and $>1$ year after SBRT, times that we considered, based on data available in the literature, as unexpected to develop radiation pneumonitis. However, we cannot assert that all cases of RRP described here are truly related to ICIs. Moreover, the RRP onset time we took into account in our study was the time of the first CT on which lung changes were seen, and depended on the frequency of the CT examination. Thus, this time could have been overestimated in some cases. Additionally, RRP is an exclusion diagnosis, this fact associated to the retrospective design of our study could potentially have led to RRP misdiagnoses. Especially, as discussed above, it could be difficult to differentiate RRP from localized ICI-related pneumonitis.

In conclusion, RRP is a delayed complication of radiotherapy triggered by immunotherapy that can be easily misdiagnosed and may occur at a higher rate than suggested in the literature. In the future, it is expected that an increasing number of cancer patients will be exposed to multiple treatment lines and treatment associations, among which radiotherapy and ICIs, leading to higher chances of developing combined toxicities, enhancing the importance of understanding and promptly recognizing them.

\section{Funding}

This study was not supported by any funding.

\section{Conflict of interest}

The authors declare that they have no conflict of interest.

There is no subject overlap with previously published work to declare.

\section{References}

[1] D’angio GJ, Farber S, Maddock CL. Potentiation of X-ray effects by actinomycin D. Radiology 1959;73:175-7.

[2] Graziani C, Hegde S, Saif MW. Radiation recall gastritis secondary to erlotinib in a patient with pancreatic cancer. Anticancer Res 2014;5.

[3] Delavan JA, Chino JP, Vinson EN. Gemcitabine-induced radiation recall myositis. Skeletal Radiol 2015;44:451-5.

[4] Jeter MD, Jänne PA, Brooks S, Burstein HJ, Wen P, Fuchs CS, et al. Gemcitabineinduced radiation recall. Int J Radiat Oncol 2002;53:394-400.

[5] Ding X, Ji W, Li J, Zhang X, Wang L. Radiation recall pneumonitis induced by chemotherapy after thoracic radiotherapy for lung cancer. Radiat Oncol 2011;6:24.

[6] Shibaki R, Akamatsu H, Fujimoto M, Koh Y, Yamamoto N. Nivolumab induced radiation recall pneumonitis after two years of radiotherapy. Ann Oncol 2017;28:1404-5.

[7] Awad R, Nott L. Radiation recall pneumonitis induced by erlotinib after palliative thoracic radiotherapy for lung cancer: case report and literature review: post radiation erlotinib induced pneumonitis. Asia Pac J Clin Oncol 2016;12:91-5.

[8] McGovern K, Ghaly M, Esposito M, Barnaby K, Seetharamu N. Radiation recall pneumonitis in the setting of immunotherapy and radiation: a focused review. Future Sci OA. 2019;5:FSO378.

[9] Chen Y, Huang Z, Xing L, Meng X, Yu J. Radiation recall pneumonitis induced by anti-PD-1 blockade: a case report and review of the literature. Front Oncol 2020;28:561.

[10] Giroux Leprieur E, Dumenil C, Julie C, Giraud V, Dumoulin J, Labrune S, et al. Immunotherapy revolutionises non-small-cell lung cancer therapy: results, perspectives and new challenges. Eur J Cancer 2017;78:16-23.

[11] Michot JM, Bigenwald C, Champiat S, Collins M, Carbonnel F, Postel-Vinay S, et al. Immune-related adverse events with immune checkpoint blockade: a comprehensive review. Eur J Cancer 2016;54:139-48.

[12] Porcu M, De Silva P, Solinas C, Battaglia A, Schena M, Scartozzi M, et al. Immunotherapy associated pulmonary toxicity: biology behind clinical and radiological features. Cancers 2019;11:305.

[13] Teng F, Li M, Yu J. Radiation recall pneumonitis induced by PD-1/PD-L1 blockades: mechanisms and therapeutic implications. BMC Med 2020;18:275.

[14] Jenkins P, Welsh A. Computed tomography appearance of early radiation injury to the lung: correlation with clinical and dosimetric factors. Int J Radiat Oncol 2011;81:97-103.

[15] Phernambucq ECJ, Palma DA, Vincent A, Smit EF, Senan S. Time and doserelated changes in radiological lung density after concurrent chemoradiotherapy for lung cancer. Lung Cancer 2011;74:451-6.

[16] Choi YW, Munden RF, Erasmus JJ, Joo Park K, Chung WK, Jeon SC, et al. Effects of radiation therapy on the lung: radiologic appearances and differential diagnosis. RadioGraphics 2004;24:985-97.

[17] Larici AR, del Ciello A, Maggi F, Santoro SI, Meduri B, Valentini V, et al. Lung abnormalities at multimodality imaging after radiation therapy for non-small cell lung cancer. RadioGraphics 2011;31:771-89.

[18] Ikezoe J, Takashima S, Morimoto S, Kadowaki K, Takeuchi N, Yamamoto T, et al. CT appearance of acute radiation-induced injury in the lung. Am J Roentgenol 1988;150:765-70.

[19] Wang S, Liao Z, Wei X, Liu HH, Tucker SL, Hu C, et al. Analysis of clinical and dosimetric factors associated with treatment-related pneumonitis (TRP) in patients with non-small-cell lung cancer (NSCLC) treated with concurrent chemotherapy and three-dimensional conformal radiotherapy (3D-CRT). Int J Radiat Oncol 2006;66:1399-407.

[20] Bernchou U, Schytte T, Bertelsen A, Bentzen SM, Hansen O, Brink C. Time evolution of regional CT density changes in normal lung after IMRT for NSCLC. Radiother Oncol 2013;109:89-94.

[21] Hanania AN, Mainwaring W, Ghebre YT, Hanania NA, Ludwig M. Radiationinduced lung injury. Chest 2019;156:150-62.

[22] Guckenberger M, Heilman K, Wulf J, Mueller G, Beckmann G, Flentje M. Pulmonary injury and tumor response after stereotactic body radiotherapy (SBRT): results of a serial follow-up CT study. Radiother Oncol 2007;85:435-42.

[23] Yamamoto T, Kadoya N, Morishita Y, Sato Y, Matsushita H, Umezawa R, et al. Assessment and agreement of the $\mathrm{CT}$ appearance pattern and its severity grading of radiation-induced lung injury after stereotactic body radiotherapy for lung cancer. Ahmad A, editor. PLOS ONE. 2018;13(10):e0204734.

[24] Trovo M, Linda A, El Naqa I, Javidan-Nejad C, Bradley J. Early and late lung radiographic injury following stereotactic body radiation therapy (SBRT). Lung Cancer 2010;69:77-85.

[25] Citrin DE, Mitchell JB. Mechanisms of normal tissue injury from irradiation. Semin Radiat Oncol 2017:27:316-24.

[26] Citrin DE, Shankavaram U, Horton JA, Shield W, Zhao S, Asano H, et al. Role of type II pneumocyte senescence in radiation-induced lung fibrosis. JNCI J Natl Cancer Inst 2013;105:1474-84.

[27] Demaria S, Golden EB, Formenti SC. Role of local radiation therapy in cancer immunotherapy. JAMA Oncol 2015;1:1325.

[28] Kainthola A, Haritwal T, Tiwari M, Gupta N, Parvez S, Tiwari M, et al. Immunological aspect of radiation-induced pneumonitis, current treatment strategies, and future prospects. Front Immunol 2017;2:506.

[29] Sharabi AB, Lim M, DeWeese TL, Drake CG. Radiation and checkpoint blockade immunotherapy: radiosensitisation and potential mechanisms of synergy. Lancet Oncol 2015;16:e498-509.

[30] Voong KR, Hazell SZ, Fu W, Hu C, Lin CT, Ding K, et al. Relationship between prior radiotherapy and checkpoint-inhibitor pneumonitis in patients with advanced non-small-cell lung cancer. Clin Lung Cancer 2019;20:e470-9.

[31] Hwang WL, Niemierko A, Hwang KL, Hubbeling H, Schapira E, Gainor JF, et al. Clinical outcomes in patients with metastatic lung cancer treated with PD-1/ PD-L1 inhibitors and thoracic radiotherapy. JAMA Oncol 2018;4:253.

[32] Shaverdian N, Lisberg AE, Bornazyan K, Veruttipong D, Goldman JW, Formenti SC, et al. Previous radiotherapy and the clinical activity and toxicity of pembrolizumab in the treatment of non-small-cell lung cancer: a secondary analysis of the KEYNOTE-001 phase 1 trial. Lancet Oncol 2017;18:895-903.

[33] Antonia SJ, Villegas A, Daniel D, Vicente D, Murakami S, Hui R, et al. Durvalumab after chemoradiotherapy in Stage III non-small-cell lung cancer. N Engl J Med 2017;377:1919-29.

[34] Cho JY, Kim J, Lee JS, Kim YJ, Kim SH, Lee YJ, et al. Characteristics, incidence, and risk factors of immune checkpoint inhibitor-related pneumonitis in patients with non-small cell lung cancer. Lung Cancer 2018;125:150-6. 
[35] Kalisz KR, Ramaiya NH, Laukamp KR, Gupta A. Immune checkpoint inhibitor therapy-related pneumonitis: patterns and management. RadioGraphics 2019;4:190036.

[36] Nishino M, Hatabu H, Hodi FS. Imaging of cancer immunotherapy: current approaches and future directions. Radiology 2019;290:9-22.
[37] Pozzessere C, Bouchaab H, Jumeau R, Letovanec I, Daccord C, Bourhis J, et al. Relationship between pneumonitis induced by immune checkpoint inhibitors and the underlying parenchymal status: a retrospective study. ERJ Open Res 2020;6:165-2019. 